\title{
New Modified Newton Type Iterative Methods
}

\author{
Jivandhar Jnawali \\ Department of Mathematics, Ratna Rajyalaxmi Campus, \\ Tribhuvan University, Kathmandu, Nepal \\ Email: jivandhar.jnawali@rrlc.tu.edu.np
}

\begin{abstract}
In this work, we present two Newton type iterative methods for finding the solution of nonlinear equations of single variable. One is obtained as variant of McDougall and Wotherspoon method, and another is obtained by amalgamation of Potra and Pta'k method and our newly introduced method. The order of convergence of these methods are $1+\sqrt{2}$ and 3.5615. Some numerical examples are given to compare the performance of these methods with some similar existing methods.
\end{abstract}

Keywords: Newton's method, Nonlinear equations, Convergence order, Iterative methods.

\section{Introduction}

Finding the solution of a single variable nonlinear equations is one of the most important tasks in numerical analysis. Nonlinear equations are frequently encountered in all fields of science and engineering. Most of the cases, it is impossible to solve such equations analytically. In those conditions, when an analytic solution cannot be obtained or the process of finding it is tedious, numerical methods are employed to get the approximate solutions. Main goal of numerical methods is to find the solution of given problem in allowed tolerance level. The construction of iterative method has been attracted the attention of mathematicians for more than four centuries. Because of the advent of different verities of computers and simulation software, the demand for numerical methods are increased rapidly in the applications to engineering and scientific fields. So during last two decades, large numbers of mathematicians are devoted to develop new numerical methods for solving nonlinear equations. One of the widely used and best known iterative methods for solving nonlinear equations is the Newton method. The iterative formula for Newton method to solve nonlinear equations $f(x)=0$ is given by [1]

$$
x_{n+1}=x_{n}-\frac{f(x)}{f^{\prime}(x)}
$$

This method converges quadratically to simple zero and need to evaluate two functions per iteration. Now days tremendous variant of this method have appeared, some of them are found in the papers [2] -[13] . In [9], McDougall and Wotherspoon proposed a new variant of Newton method using a different technique. Their method's iterative scheme is as follows:

If $x_{0}$ is the initial approximation, then

$$
\begin{aligned}
& x_{0}^{*}=x_{0} \\
& x_{1}=x_{0}-\frac{f\left(x_{0}\right)}{f^{\prime}\left(\frac{x_{0}+x_{0}^{*}}{2}\right)}=x_{0}-\frac{f\left(x_{0}\right)}{f^{\prime}\left(x_{0}\right)} .
\end{aligned}
$$

Subsequently for $n \geq 1$, the iteration can be obtained as

$$
\begin{aligned}
& x_{n}^{*}=x_{n}-\frac{f\left(x_{n}\right)}{f^{\prime}\left(\frac{x_{n-1}+x_{n-1}^{*}}{2}\right)} \\
& x_{n}=x_{n}-\frac{f\left(x_{n}\right)}{f^{\prime}\left(\frac{x_{n}+x_{n}^{*}}{2}\right)} .
\end{aligned}
$$


This method is a predictor-corrector type method with convergence order $1+\sqrt{2}$. In this method, we have to evaluate two functions per iteration as in Newton's method but we calculate the derivative at some convenient point instead of previously iterated point.

\section{The Methods}

Here, we suggest the following method as a variant of method (2a)-(2d) by using harmonic mean instead of arithmetic mean. If $x_{0}$ is the initial approximation, then

$$
\begin{aligned}
& x_{0}^{*}=x_{0} \\
& x_{1}=x_{0}-\frac{f\left(x_{0}\right)}{f^{\prime}\left(\frac{2 x_{0} x_{0}^{*}}{x_{0}+x_{0}^{*}}\right)}=x_{0}-\frac{f\left(x_{0}\right)}{f^{\prime}\left(x_{0}\right)} .
\end{aligned}
$$

Subsequently for $n \geq 1$, the iteration can be obtained as

$$
\begin{aligned}
& x_{n}^{*}=x_{n}-\frac{f\left(x_{n}\right)}{f^{\prime}\left(\frac{2 x_{n-1} x_{n-1}^{*}}{x_{n-1}+x_{n-1}^{*}}\right)} \\
& x_{n}=x_{n}-\frac{f\left(x_{n}\right)}{f^{\prime}\left(\frac{2 x_{n} x_{n}^{*}}{x_{n}+x_{n}^{*}}\right)} .
\end{aligned}
$$

The third order method obtained by Potra and Pta'k in [ 11 ] is given by

$$
x_{n+1}=x_{n}-\frac{f\left(x_{n}-\frac{f\left(x_{n}\right)}{f^{\prime}\left(x_{n}\right)}\right)+f\left(x_{n}\right)}{f^{\prime}\left(x_{n}\right)} .
$$

Here, we suggest variant of method (4) whose iterative scheme is as follows:

If $x_{0}$ is the initial approximation, then

$$
\begin{gathered}
x_{0}^{*}=x_{0} \\
x_{1}=x_{0}-\frac{f\left[x_{0}-\frac{f\left(x_{0}\right)}{f^{\prime}\left(\frac{2 x_{0} x_{0}^{*}}{x_{0}+x_{0}^{*}}\right)}\right]+f(0)}{f^{\prime}\left(\frac{2 x_{0} x_{0}^{*}}{x_{0}+x_{0}^{*}}\right)}=x_{0}-\frac{f\left[x_{0}-\frac{f\left(x_{0}\right)}{f^{\prime}\left(x_{0}\right)}\right]+f\left(x_{0}\right)}{f^{\prime}\left(x_{0}\right)}
\end{gathered}
$$

Subsequently, for $n \geq 1$, the iterations can be obtained as below:

$$
\begin{gathered}
x_{n}^{*}=x_{n}-\frac{f\left[x_{n}-\frac{f\left(x_{n}\right)}{f^{\prime}\left(\frac{2 x_{n-1} x_{n-1}^{*}}{x_{n-1}+x_{n-1}^{*}}\right)}\right]+f\left(x_{n}\right)}{f^{\prime}\left(\frac{2 x_{n-1} x_{n-1}^{*}}{x_{n-1}+x_{n-1}^{*}}\right)} \\
=x_{n}-\frac{f\left[x_{n}-\frac{f\left(x_{n}\right)}{f^{\prime}\left(\frac{2 x_{n} x_{n}^{*}}{x_{n}+x_{n}^{*}}\right)}\right]+f\left(x_{n}\right)}{f^{\prime}\left(\frac{2 x_{n} x_{n}^{*}}{x_{n}+x_{n}^{*}}\right)}
\end{gathered}
$$

\section{Convergence Analysis}

For the convergence of the method (3a)-(3d), we prove the following results.

Theorem 3.1 Let $\alpha$ be a simple zero of a function $f$ which has sufficient number of smooth derivatives in a neighborhood of $\alpha$. Then for solving the nonlinear equation $f(x)=0$, the method (3a)-(3d) is convergent with order of convergence $1+\sqrt{2}$.

\section{Proof:}

Suppose $e_{n}^{*}$ and $e_{n}$ denote the errors in the iterates $x_{n}$ and $x_{n}^{*}$ respectively and 
$c_{k}=\frac{f^{k}(\alpha)}{k ! f^{\prime}(\alpha)}, k=2,3,4 \ldots$. Then the error equation of the Newton's method (1) is given by [1 ]

$$
e_{n+1}=c_{2} e_{n}^{2}
$$

where the terms involving higher power of $e_{n}$ are ignored. Next, we proceed the analysis of convergence of method (3a)-(3d). Clearly, $e_{0}^{*}=e_{0}$ and from view (6), the error equation for (3b) is given by

$$
e_{1}=c_{2} e_{0}^{2}
$$

Using this, the Taylor series expansion, and binomial expansion, the error in $x_{1}^{*}$ is given by

$$
\begin{aligned}
e_{1}^{*} & =e_{1}-\frac{f\left(\alpha+e_{1}\right)}{f \prime\left(\alpha+e_{0}\right)}=e_{1}-\frac{e_{1}+c_{2} e_{1}^{2}+c_{3} e_{1}^{3}+O\left(e_{0}^{4}\right)}{1+2 c_{2} e_{0}+3 c_{3} e_{0}^{2}+O\left(e_{0}^{3}\right)} \\
& =e_{1}-\left(e_{1}+c_{2} e_{1}^{2}+c_{3} e_{1}^{3}+O\left(e_{0}^{4}\right)\right)\left(1-2 c_{2} e_{0}-3 c_{3} e_{0}^{2}-4 c_{2}^{2} e_{0}^{2}+O\left(e_{0}^{3}\right)\right) \\
& =2 c_{2} e_{0} e_{1} \\
& =2 c_{2}^{2} e_{0}^{3}
\end{aligned}
$$

where the terms involving higher power of $e_{0}$ are neglected.

Next,

$$
\frac{2 x_{1} x_{1}^{*}}{x_{1}+x_{1}^{*}}=\frac{2\left(\alpha+e_{1}\right)\left(\alpha+e_{1}^{*}\right)}{\left(\alpha+e_{1}\right)+\left(\alpha+e_{1}^{*}\right)}=\left(\alpha+e_{1}+e_{1}^{*}+\frac{e_{1} e_{1}^{*}}{\alpha}\right)\left(1+\frac{e_{1}+e_{1}^{*}}{2 \alpha}\right)^{-1}=\alpha+\frac{e_{1}+e_{1}^{*}}{2},
$$

where the terms involving higher powers of $e_{1}$ and $e_{1}^{*}$ are neglected. Therefore, the error equation for (3d) with $n=1$ and using (7) and (8) is given by

$$
\begin{aligned}
e_{2}=e_{1}-\frac{f\left(\alpha+e_{1}\right)}{f^{\prime}\left(\alpha+\frac{e_{1}+e_{1}^{*}}{\alpha}\right)} & =e_{1}-\left(e_{1}+c_{2} e_{1}^{2}+c_{3} e_{1}^{3}\right)\left[1+2 c_{2}\left(\frac{e_{1}+e_{1}^{*}}{2}\right)+3 c_{3}\left(\frac{e_{1}+e_{1}^{*}}{2}\right)^{2}\right]^{-1} \\
& =c_{2} e_{1} e_{1}^{*}=2 c_{2}^{4} e_{0}^{5}
\end{aligned}
$$

In general, it can be shown that for $n \geq 2$, the errors in $x_{n}^{*}$ and $x_{n}$ can be obtained recursively by the relations

and

$$
\begin{gathered}
e_{n}^{*}=c_{2} e_{n} e_{n-1} \\
e_{n+1}=c_{2} e_{n} e_{n}^{*}
\end{gathered}
$$

In order to find the order of convergence of the method, we need a relation of the form

$$
e_{n+1}=K e_{n}^{p}
$$

where $K$ is some constant. Thus,

$$
e_{n}=K e_{n-1}^{p} \quad \text { or } \quad e_{n-1}=K^{-\frac{1}{p}} e_{n}^{\frac{1}{p}}
$$

From (10), (11), (12) and (13), we have

$$
K e_{n}^{p}=K c_{2} e_{n} e_{n}^{*}=K c_{2} e_{n} c_{2} e_{n} e_{n-1}=K^{1-\frac{1}{p}} C_{2}^{2} e_{n}^{2+\frac{1}{p}}
$$

Equating the power of $e_{n}$,

$$
\begin{aligned}
p=2+\frac{1}{p} \\
\therefore p=1+\sqrt{2}
\end{aligned}
$$

Hence the method (3a)-(3d) is convergent with order of convergence $1+\sqrt{2}$.

Theorem 3.2 Let $\alpha$ be a simple zero of a function $f$ which has sufficient number of smooth derivatives in a neighborhood of $\alpha$. Then for solving the nonlinear equation $f(x)=0$, the method (5a)-(5d) is convergent with order of convergence 3.5615 


\section{Jivandhar Jnawali / New Modified Newton Type Iterative Methods}

\section{Proof:}

Let $e_{n}$ and $e_{n}^{*}$ denote, respectively, the errors in $x_{n}$ and $x_{n}^{*}$. Also, we denote $c_{k}=\frac{f^{k}(\alpha)}{k ! f^{\prime}(\alpha)}, k=$ $2,3,4, \ldots . .$, which are constants. Then from (5a), $x_{0}^{*}=x_{0}$ implies $e_{0}^{*}=e_{0}$. Next, we proceed to calculate the error $e_{1}$ in $x_{1}$. By using Taylor series expansion and binomial expansion, we get

$$
\begin{aligned}
x_{0}-\frac{f\left(x_{0}\right)}{f^{\prime}\left(x_{0}\right)} & =\alpha+e_{0}-\frac{f\left(\alpha+e_{0}\right)}{f^{\prime}\left(\alpha+e_{0}\right)}=\alpha+e_{0}-\frac{e_{0}+c_{2} e_{0}^{2}+c_{3} e_{0}^{3}+O\left(e_{0}^{4}\right)}{1+2 c_{2} e_{0}+3 c_{3} e_{0}^{2}+O\left(e_{0}^{3}\right)} \\
& =\alpha+e_{0}-\left[e_{0}+c_{2} e_{0}^{2}+c_{3} e_{0}^{3}+O\left(e_{0}^{4}\right)\right]\left[1+2 c_{2} e_{0}+3 c_{3} e_{0}^{2}+O\left(e_{0}^{3}\right)\right]^{-1} \\
& =\alpha+c_{2} e_{0}^{2}+\left(2 c_{3}-2 c_{2}^{2}\right) e_{0}^{3}+O\left(e_{0}^{4}\right),
\end{aligned}
$$

So that after some calculations, we get

$$
\begin{gathered}
f\left(x_{0}-\frac{f\left(x_{0}\right)}{f^{\prime}\left(x_{0}\right)}\right)=f^{\prime}(\alpha)\left[c_{2} e_{0}^{2}+\left(2 c_{3}-2 c_{2}^{2}\right) e_{0}^{3}+c_{2}^{3} e_{0}^{4}+O\left(e_{0}^{5}\right)\right] \\
f\left(x_{0}-\frac{f\left(x_{0}\right)}{f^{\prime}\left(x_{0}\right)}\right)+f\left(x_{0}\right)=f^{\prime}(\alpha)\left[e_{0}+2 c_{2} e_{0}^{2}+3 c_{3} e_{0}^{3}-2 c_{2}^{2} e_{0}^{3}+c_{2}^{3} e_{0}^{4}+O\left(e_{0}^{5}\right)\right]
\end{gathered}
$$

and

$$
\begin{aligned}
\frac{f\left(x_{0}-\frac{f\left(x_{0}\right)}{f^{\prime}\left(x_{0}\right)}\right)+f\left(x_{0}\right)}{f^{\prime\left(x_{0}\right)}} & =\left[e_{0}+2 c_{2} e_{0}^{2}+3 c_{3} e_{0}^{3}-2 c_{2}^{2} e_{0}^{3}+c_{2}^{3} e_{0}^{4}+O\left(e_{0}^{5}\right)\right]\left[1+2 c_{2} e_{0}+3 c_{3} e_{0}^{2}+O\left(e_{0}^{3}\right)\right]^{-1} \\
& =e_{0}-2 c_{2}^{2} e_{0}^{3}+O\left(e_{0}^{4}\right)
\end{aligned}
$$

Thus from (5b),

$$
\begin{aligned}
\alpha+e_{1} & =\alpha+e_{0}-e_{0}+2 c_{2}^{2} e_{0}^{3}+O\left(e_{0}^{4}\right) \\
\therefore \quad e_{1} & =a e_{0}^{3},
\end{aligned}
$$

where $a=2 c_{2}^{2}$ and the terms involving higher power of $e_{n}$ are neglected. Again, from (5c)

$$
x_{1}^{*}=x_{1}-\frac{f\left[x_{1}-\frac{f\left(x_{1}\right)}{f^{\prime}\left(x_{0}\right)}\right]+f\left(x_{1}\right)}{f^{\prime}\left(x_{0}\right)}
$$

Here $f\left[x_{1}-\frac{f\left(x_{1}\right)}{f^{\prime}\left(x_{0}\right)}\right]=f\left[\alpha+e_{1}-\frac{f\left(\alpha+e_{1}\right)}{f^{\prime}\left(\alpha+e_{0}\right)}\right]=f\left[\alpha+e_{1}-\frac{e_{1}+c_{2} e_{1}^{2}+c_{3} e_{1}^{3}+O\left(e_{1}^{4}\right)}{1+2 c_{2} e_{0}+3 c_{3} e_{0}^{2}+O\left(e_{0}^{3}\right)}\right]$

After some calculation, we get

$$
=f^{\prime}(\alpha)\left[1+2 c_{2} e_{0} e_{1}+3 c_{3} e_{0}^{2} e_{1}-4 c_{2}^{2} e_{0}^{2} e_{1}+O\left(e_{0}^{6}\right)\right]
$$

Also,

$$
f\left[x_{1}-\frac{f\left(x_{1}\right)}{f^{\prime}\left(x_{0}\right)}\right]+f\left(x_{1}\right)=f^{\prime}(\alpha)\left[e_{1}+2 c_{2} e_{0} e_{1}+3 c_{3} e_{0}^{2} e_{1}-4 c_{2}^{2} e_{0}^{2} e_{1}+\cdots\right]
$$

and

$$
\begin{aligned}
\frac{f\left[x_{1}-\frac{f\left(x_{1}\right)}{\left.f^{\prime}\left(x_{0}\right)\right]}\right]+f\left(x_{1}\right)}{f^{\prime}\left(x_{0}\right)} & =\left[e_{1}+2 c_{2} e_{0} e_{1}+3 c_{3} e_{0}^{2} e_{1}-4 c_{2}^{2} e_{0}^{2} e_{1}+\cdots\right]\left[1+2 c_{2} e_{0}+3 c_{3} e_{0}^{2}+\cdots\right]^{-1} \\
& =e_{1}-4 c_{2}^{2} e_{0}^{2} e_{1}+O\left(e_{0}^{4}\right)
\end{aligned}
$$

Thus the error $e_{1}^{*}$ in $x_{1}^{*}$ is given by

$$
e_{1}^{*}=e_{1}-\left[e_{1}-4 c_{2}^{2} e_{0}^{2} e_{1}+O\left(e_{0}^{4}\right)\right]=a b e_{0}^{5}
$$

where $b=4 c_{2}^{2}$ and the terms involving higher power of $e_{0}$ are neglected.

Next, we compute the error $e_{2}$ in $x_{2}$.

Now 


$$
\begin{aligned}
\frac{f\left(x_{1}\right)}{f^{\prime}\left(\frac{2 x_{1} x_{1}^{*}}{x_{1}+x_{1}^{*}}\right)} & =\frac{f^{\prime}(\alpha)\left(e_{1}+c_{2} e_{1}^{2}+c_{3} e_{1}^{3}+O\left(e_{1}^{4}\right)\right)}{f^{\prime}\left(\alpha+\frac{e_{1}+e_{1}^{*}}{2}\right)}=\frac{e_{1}+c_{2} e_{1}^{2}+c_{3} e_{1}^{3}+O\left(e_{1}^{4}\right)}{1+c_{2} e_{1}+c_{2} e_{1}^{*}+\frac{3}{4} c_{3} e_{1}^{2}+O\left(e_{0}^{3}\right)} \\
& =e_{1}+\frac{1}{4} c_{3} e_{1}^{3}-c_{2} e_{1} e_{1}^{*}-c_{2}^{2} e_{1}^{2} e_{1}^{*}+\cdots \\
\therefore x_{1} & -\frac{f\left(x_{1}\right)}{f^{\prime}\left(\frac{2 x_{1} x_{1}^{*}}{x_{1}+x_{1}^{*}}\right)}=\alpha-\frac{1}{4} c_{3} e_{1}^{3}+c_{2} e_{1} e_{1}^{*}+c_{2}^{2} e_{1}^{2} e_{1}^{*}+\cdots
\end{aligned}
$$

where the higher power terms are neglected. Thus

and

$$
f\left(x_{1}-\frac{f\left(x_{1}\right)}{f^{\prime}\left(\frac{2 x_{1} x_{1}^{*}}{x_{1}+x_{1}^{*}}\right)}\right)=f^{\prime}(\alpha)\left(c_{2} e_{1} e_{1}^{*}+c_{2}^{2} e_{1}^{2} e_{1}^{*}-\frac{1}{4} c_{3} e_{1}^{3} \ldots\right)
$$

$$
\begin{gathered}
f\left(x_{1}-\frac{f\left(x_{1}\right)}{f^{\prime}\left(\frac{2 x_{1} x_{1}^{*}}{x_{1}+x_{1}^{*}}\right)}\right)+f\left(x_{1}\right)=e_{1} f^{\prime}(\alpha)\left(1+c_{2} e_{1}+c_{3} e_{1}^{2}+c_{2} e_{1}^{*}+c_{2}^{2} e_{1} e_{1}^{*}-\frac{1}{4} c_{3} e_{1}^{3}+\cdots\right) . \\
\frac{f\left(x_{1}-\frac{f\left(x_{1}\right)}{f^{\prime}\left(\frac{2 x_{1} x_{1}^{*}}{x_{1}+x_{1}^{*}}\right)}\right)+f\left(x_{1}\right)}{f^{\prime}\left(\frac{2 x_{1} x_{1}^{*}}{x_{1}+x_{1}^{*}}\right)}=e_{1}-\frac{3}{2} c_{3} e_{1}^{2} e_{1}^{*} \ldots
\end{gathered}
$$

From (5d),

$$
x_{2}=x_{1}-\frac{f\left(x_{1}-\frac{f\left(x_{1}\right)}{f^{\prime}\left(\frac{2 x_{1} x_{1}^{*}}{x_{1}+x_{1}^{*}}\right)}\right)+f\left(x_{1}\right)}{f^{\prime}\left(\frac{2 x_{1} x_{1}^{*}}{x_{1}+x_{1}^{*}}\right)}
$$

So, after substituting the values, we get

$$
\begin{gathered}
\alpha+e_{2}=\alpha+e_{1}-\left(e_{1}-\frac{3}{2} c_{3} e_{1}^{2} e_{1}^{*}+\cdots\right) \\
\therefore e_{2}=\frac{3}{2} c_{3} e_{1}^{2} e_{1}^{*}=a^{3} b c e_{0}^{11},
\end{gathered}
$$

Where $c=\frac{3}{2} \quad c_{3}$. In fact it can be worked out for $n \geq 1$, that the following relation holds:

$$
e_{n+1}=c e_{n}^{2} e_{n}^{*}
$$

To compute $e_{n+1}$ explicitly, we need $e_{n}^{*}$. We already obtained the value of $e_{1}^{*}$ and next we compute $e_{2}^{*}$. From $(5 \mathrm{~d})$

$$
x_{2}^{*}=x_{2}-\frac{f\left[x_{2}-\frac{f\left(x_{2}\right)}{f^{\prime}\left(\frac{2 x_{1} x_{1}^{*}}{x_{1}+x_{1}^{*}}\right)}\right]+f\left(x_{n}\right)}{f^{\prime}\left(\frac{2 x_{1} x_{1}^{*}}{x_{1} x_{1}^{*}}\right)}
$$

Proceed stepwise as above, the error $e_{2}^{*}$ in $x_{2}^{*}$ is given by the relation

$$
e_{2}^{*}=d e_{1}^{2} e_{2}
$$

Where $d=c_{2}^{2}$ and, it can be checked that, in general, for $n \geq 2$, the following relation holds:

$$
e_{n}^{*}=d e_{n-1}^{2} e_{n}
$$


From (15) and (16), we conclude that the errors $e_{n}^{*}$ in $x_{n}^{*}$ and $e_{n+1}$ in $x_{n+1}$ for $n \geq 2$ in method (5a)(5d) satisfy the recursion formula given below:

$$
\begin{gathered}
e_{n}^{*}=d e_{n-1}^{2} e_{n} \\
e_{n+1}=c e_{n}^{2} e_{n}^{*}
\end{gathered}
$$

To obtained convergence order of the method, we need a relation of the form

where $\mathrm{K}$ is some constant. Thus,

$$
e_{n+1}=K e_{n}^{p}
$$

$$
e_{n}=K e_{n-1}^{p} \quad \text { or } \quad e_{n-1}=K^{-\frac{1}{p}} e_{n}^{\frac{1}{p}}
$$

From (17), (18), (19) and (20),

$$
K e_{n}^{p}=c e_{n}^{2} e_{n}^{*}=c e_{n}^{2} d e_{n-1}^{2} e_{n}=c d K^{-\frac{2}{p}} e_{n}^{3+\frac{2}{p}}
$$

Equating the power of $e_{n}$,

$$
p=3+\frac{2}{p} \quad \therefore p=\frac{3 \pm \sqrt{17}}{2}
$$

Hence the method (5a)-(5d) is convergent with convergence order $\frac{3+\sqrt{17}}{2} \cong 3.5615$.

\section{Numerical Experiments}

In order to check the performance of the introduced methods (3a)-(3d) and (5a)-(5d), we exhibit the numerical results on some nonlinear equations of single variable. We also compare the results of these methods with Newton method (NM), McDougall and Wotherspoon (MW) method, and Potra and Pta'k method. Numerical computations have been performed using the Matlab software. We use the stopping criteria $\left|x_{n+1}-x_{n}\right|<(10)^{-12}$ or $\left|x_{n+1}\right|<(10)^{-14}$ for the iterative process of our results.

For the numerical examples, we use following text functions and their roots $\alpha$

(i) $f_{1}=(x-2)^{23}-1, \quad \alpha=3$

(ii) $f_{2}=\cos x-x e^{x}+x^{2}, \quad \alpha=0.639154069332008$

(iii) $f_{3}=x^{6}-x-1, \quad \alpha=1.134724138401519$

Table 1: $f_{1}=(x-2)^{23}-1$, and initial guess $x=4$

\begin{tabular}{|c|l|l|l|l|l|}
\hline $\begin{array}{l}\text { No. of } \\
\text { Iterations }\end{array}$ & Newton Method & M-W Method & $\begin{array}{l}\text { Newly introduced } \\
\text { Method (3a)-(3d) }\end{array}$ & $\begin{array}{l}\text { Potra and Pta'k } \\
\text { Method }\end{array}$ & $\begin{array}{l}\text { Present Method } \\
(5 \mathrm{a})-(5 \mathrm{~d})\end{array}$ \\
\hline 1 & 3.913043488626895 & 3.913043488626895 & 3.913043488626895 & 3.881762281757005 & 3.881762281757005 \\
2 & 3.829867712336497 & 3.813403617069735 & 3.813331074452638 & 3.770514691012868 & 3.751168249608405 \\
3 & 3.750308319871752 & 3.719262465432603 & 3.719132009936569 & 3.665844113356311 & 3.629953250636192 \\
4 & 3.674208153011303 & 3.629998067126574 & 3.629817244700443 & 3.567362235341004 & 3.517123737847595 \\
5 & 3.601417012345112 & 3.545369541447942 & 3.545145511948340 & 3.474705145072179 & 3.412115738950753 \\
6 & 3.531791563484456 & 3.465137701182709 & 3.464876921225920 & 3.387535965688113 & 3.314417477331388 \\
7 & 3.465195593647396 & 3.389080467269836 & 3.388788790381042 & 3.305558823234900 & 3.223669633399193 \\
8 & 3.401501177624357 & 3.317001067351732 & 3.316683886407281 & 3.228575744377471 & 3.140096774314826 \\
9 & 3.340592238813068 & 3.248757430234993 & 3.248420046055467 & 3.156705026488715 & 3.066464605301569 \\
\hline
\end{tabular}


Nepal Journal of Mathematical Sciences (NJMS), Vol.2(1) , 2021 (February): 17-24

\begin{tabular}{|l|l|l|l|l|l|}
\hline 10 & 3.282374444500791 & 3.184357717630727 & 3.184006488699039 & 3.091180490016622 & 3.014477358683509 \\
11 & 3.226801820893740 & 3.124261554464694 & 3.123907609777858 & 3.036856948205285 & 3.000181100048933 \\
12 & 3.173947045831000 & 3.070261667428139 & 3.069931829367179 & 3.005553045154960 & 3.000000000030649 \\
13 & 3.124182323440681 & 3.027446469454418 & 3.027203785176629 & 3.000036207524484 & 3.000000000000000 \\
14 & 3.078615102622522 & 3.004438034641327 & 3.004352969040154 & 3.000000000011477 & \\
15 & 3.039945182614113 & 3.000074769586678 & 3.000071395956669 & 3.000000000000000 & \\
16 & 3.013097347814351 & 3.000000003879636 & 3.000000003465490 & & \\
17 & 3.001704377684283 & 3.000000000000000 & 3.000000000000000 & & \\
18 & 3.000031522843926 & & & & \\
19 & 3.000000010927831 & & & & \\
20 & 3.000000000000001 & & & & \\
21 & 3.000000000000000 & & & & \\
\end{tabular}

Table 2: $f_{2}=\cos x-x e^{x}+x^{2}$ and initial guess $x=2$

\begin{tabular}{|c|l|l|l|l|l|}
\hline $\begin{array}{l}\text { No. of } \\
\text { Iterations }\end{array}$ & Newton Method & M-W Method & $\begin{array}{l}\text { Present Method } \\
(3 \mathrm{a})-(3 \mathrm{~d})\end{array}$ & $\begin{array}{l}\text { Potra and Pta'k } \\
\text { Method }\end{array}$ & $\begin{array}{l}\text { Present Method } \\
(5 \mathrm{a})-(5 \mathrm{~d})\end{array}$ \\
\hline 1 & 1.413190095241312 & 1.413190095241312 & 1.413190095241312 & 1.221708204826725 & 1.221708204826725 \\
2 & 0.960950362222547 & 0.890336187671187 & 0.884765708414379 & 0.744561133808421 & 0.698655987801723 \\
3 & 0.708489670672238 & 0.664695575326538 & 0.662363993421890 & 0.640375324008681 & 0.639188120754996 \\
4 & 0.642803431695474 & 0.639281518751900 & 0.639251779238558 & 0.639154098569363 & 0.639154096332008 \\
5 & 0.639164526016085 & 0.639154096646505 & 0.639154096497285 & 0.639154096332007 & \\
6 & 0.639154096417341 & 0.639154096332008 & 0.639154096332008 & & \\
7 & 0.639154096332008 & & & & \\
\end{tabular}

Table 3: $f_{3}=x^{6}-x-1$ and initial guess $x=2$

\begin{tabular}{|c|l|l|l|l|l|}
\hline $\begin{array}{l}\text { No. of } \\
\text { Iterations }\end{array}$ & Newton Method & M-W Method & $\begin{array}{l}\text { Present Method } \\
(3 \mathrm{a})-(3 \mathrm{~d})\end{array}$ & $\begin{array}{l}\text { Potra and Pta'k } \\
\text { Method }\end{array}$ & $\begin{array}{l}\text { Present Method } \\
(5 \mathrm{a})-(5 \mathrm{~d})\end{array}$ \\
\hline 1 & 1.680628272251309 & 1.680628272251309 & 1.680628272251309 & 1.576686172124548 & 1.576686172124548 \\
2 & 1.430738988239062 & 1.387614413767816 & 1.386095807015648 & 1.287827012394893 & 1.249344732145450 \\
3 & 1.254970956109436 & 1.205999615828861 & 1.204636665057341 & 1.152665486084360 & 1.138755278835567 \\
4 & 1.161538432773313 & 1.140433658814203 & 1.140138942841691 & 1.134784110035479 & 1.134724181083601 \\
5 & 1.136353274170505 & 1.134740819036991 & 1.134738745696497 & 1.134724138404039 & 1.134724138401520 \\
6 & 1.134730528343629 & 1.134724138412645 & 1.134724138409576 & 1.134724138401520 & \\
7 & 1.134724138500221 & 1.134724138401519 & 1.134724138401519 & & \\
8 & 1.134724138401519 & & & & \\
\hline
\end{tabular}




\section{Conclusion}

In this work, we have presented two new Newton type iterative methods having convergence order $1+\sqrt{2}$ and 3.5615 for solving nonlinear equations of single variable. From the numerical as well as theoretical result, the newly introduced method (3a)-(3d) whose order of convergence and efficiency index are higher than Newton's methods and same with McDougall and Wotherspoon method (2a)-(2d). Also hybrid method (5a)-(5d) is converge to the root faster than Potra and Pta'k method but in this method we have to evaluate one more function after first iteration.

\section{References}

[1]. Bradie, B. (2007). A Friendly Introduction to Numerical Analysis. Pearson Education Inc.

[2]. Dheghain, M. and Hajarian, M. (2010). New iterative method for solving nonlinear equations fourthorder Convergence. International Journal of Computer Mathematics. 87: 834-839.

[3]. Homeier, H. H. H. (2005). On Newton's type methods with cubic convergence. J. Comput. Appl. Math. 176: 425-432.

[4]. Jain, D. (2013). Families of Newton-like methods with fourth-order convergence. International Journal of Computer Mathematics. 90: 1072-1082.

[5]. Jain, P., Bhatta C.R. and Jnawali,, J. (2015) Modified Newton type methods with higher order of convergence. Jordan J. Math. Stat. 8(4): 327-341.

[6]. Jain, P., Bhatta C.R. and Jnawali,, J. (2016). Newton type iterative methods with higher order of convergence, J. Numer. Anal. Approx. Theory. 45: 14-26.

[7]. Jnawali, J. and Bhatta, C. R.(2018). Two higher order iterative methods for solving nonlinear equations, Journal of the Institute of Engineering. 14: 179-18.

[8]. Jnawali, J. (2020). Some iterative methods to solve nonlinear equations with faster convergence. Punjab University Journal of Mathematics. 52(2): 57-72.

[9]. McDougall T. J. and Wotherspoon, S. J. (2014). A simple modification of Newton's method to achieve convergence of order $1+\sqrt{2}$. Appl. Math. Letter. 29: 20-25.

[10]. Özban, A.Y. (2004) Some new variants of Newton's method. Applied Mathematics Letters. 13: $677-682$.

[11]. Potra, F.A. and Pt'ak, V. (1984). Nondecrete introduction and iterative processes. Research Note in Mathematics. Vol.203 Pitman, Bostan.

[12]. Wang, P. (2011). A third order family of Newton like iteration method for solving nonlinear equation. Journal of Numerical Mathematics and Stochastics. 3 : 11-19.

[13]. Weerakoon, S. and Fernando, T.G.I. (2002). A variant of Newton's method with accelerated thirdorder convergence. Applied Mathematics Letters. 13 : 87-93. 